\title{
Точно решаемая модель графеновой наноленты с зигзагообразными краями
}

\author{
(C) С.Ю. Давыдов ${ }^{1}$, А.В. Зубов ${ }^{2}$ \\ ${ }^{1}$ Физико-технический институт им. А.Ф. Иоффе Российской академии наук, \\ 194021 Санкт-Петербург, Россия \\ ${ }^{2}$ Санкт-Петербургский национальный исследовательский университет \\ информационных технологий, механики и оптики (ИТМО), \\ 197101 Санкт-Петербург, Россия \\ E-mail: Sergei_Davydov@mail.ru \\ Поступила в Редакцию 1 апреля 2019 г. \\ В окончательной редакции 8 октября 2019 г. \\ Принята к публикации 15 октября 2019 г.
}

В рамках простой структурной модели определены точные аналитические выражения для локальных плотностей состояний и чисел заполнения атомов зигзагообразной кромки наноленты графена. В качестве примера применения полученных результатов рассмотрена задача о точечном дефекте Костера-Слэтера.

Ключевые слова: графеновая нанолента, зигзагообразная кромка, точечный дефект.

DOI: 10.21883/FTP.2020.02.48913.9125

\section{1. Введение}

Интерес к фрагментам бесконечного листа графена, таким как наноленты $[1,2]$ и двумерные углеродные островки [3], обусловлен как с фундаментальной, так и с прикладной точек зрения. В первом случае это связано с появлением (и проявлением) особых граничных состояний, во втором - с возможностью использования таких нанообъектов в приборных структурах. Отметим, что подавляющее число теоретических работ по углеродным наноструктурам (как, впрочем, и во всей физике конденсированного состояния) представляет собой численные расчеты в рамках DFT (density functional theory) [4-6]. На этом фоне построение простых моделей, допускающих, в идеале, точные решения, по-прежнему представляется полезным в первую очередь для выявления главных определяющих факторов проблемы, например: механизмов того или иного явления или основных вкладов в вычисляемое значение какой-либо физической характеристики (см. заключительный абзац нобелевской речи Ф. Андерсона [7]). В настоящей работе предлагается модель графеновой наноленты с зигзагообразными кромками, допускающая точное решение.

\section{2. Энергетические зоны и плотности состояний}

Для построения структурной модели графеновой наноленты, представленной на рис. 1, мы использовали двумерную решетку типа кирпичной стенки $[8,9]$, топологически эквивалентную решетке графена. Атомы типа $-11,11,-1-1,1-1$, имеющие двух ближайших соседей (б.с.), имитируют внешние атомы зигзагообразной кромки; атомы типа 01 и 0-1, имеющие трех б.с., представляют внутренние атомы наноленты. Такой под- ход уже использовался нами в задаче о декорировании зигзагообразных кромок графеновой наноленты [10].

Представим функцию Грина, отвечающую изолированному атому углерода, в виде $g^{-1}(\omega)=\omega-\varepsilon+i s$, где $\omega-$ текущая энергия, $\varepsilon-$ энергия состояния $\left|p_{z}\right\rangle$ атома углерода, в дальнейшем принимаемая за начало отсчета (точка Дирака), $s=0^{+}$. Включая взаимодействия $t$ между б.с. в наноленте и воспользовавшись результатами работы [10], получим соответственно для внешних и внутренних атомов функции Грина вида:

$$
\begin{gathered}
G_{01,01} \equiv G_{0}(\omega, k)=g \frac{1-g^{2} t^{2} \Phi(k)}{D(\omega, k)}, \\
G_{11,11} \equiv G_{1}(\omega, k)=g \frac{1-g^{2} t^{2}[1+\Phi(k)]}{D(\omega, k)}, \\
D(\omega, k)=\left[1-g^{2} t^{2} \Phi(k)\right]^{2}-g^{2} t^{2},
\end{gathered}
$$

где $\Phi(k)=4 \cos ^{2}(k a), a-$ расстояние между б.с. в графене, $|k| \leq \pi / 2$ волновой вектор. Электронный

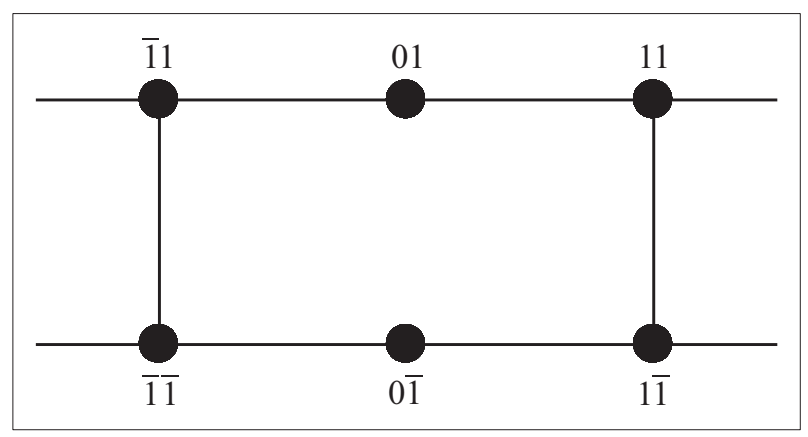

Рис. 1. Структурная модель наноленты. Атомы типа -11, 11, -1-1, 1-1 представляют внутренние атомы зигзагообразной кромки; атомы типа 01 и 0-1 - внешние атомы наноленты. 
спектр наноленты, определяемый полюсами функций Грина (1) и (2), т.е. корнями уравнения $D(\omega, k)=0$, имеет вид

$$
\omega_{p}(k)= \pm t[\sqrt{1+4 \Phi(k)} \pm 1] / 2,
$$

где индекс $p$ нумерует зоны. Условимся, что нумерация $p$-зон идет в сторону убывания энергии: $p=1$ соответствует знакам плюс перед квадратной скобкой и в квадратной скобке, $p=2$ - знакам плюс и минус, $p=3$ - знакам минус в обоих случаях, $p=4-$ знакам минус и плюс. Две верхние зоны, отвечающие положительной энергии, представлены на рис. 2. Из (4) следует, что $\omega_{1}(0)=-\omega_{4}(0)=t(\sqrt{17}+1) / 2 \approx 2.56 t$, $\omega_{2}(0)=-\omega_{3}(0)=t(\sqrt{17}-1) / 2 \approx 1.56 t, \quad \omega_{1}(\pi / 2 a)=$ $=-\omega_{4}(\pi / 2 a)=t, \omega_{2}(\pi / 2 a)=-\omega_{3}(\pi / 2 a)=0$.

Следует подчеркнуть, что для рассматриваемой здесь двухцепочечной наноленты $(n=2)$ ширина области сплошного спектра равна $W_{2}=2 \omega_{1}(0) \approx 5.12 t$, тогда как для бесконечного листа графена ширина этой области $W_{\infty}=6 t$. Ясно, что, включая в модель дополнительные внутренние цепочки, будем переходить от $W_{2}$ к $W_{n}$.

Для нахождения энергетической плотности состояний необходимо вычислить зависящие только от энергии функции Грина

$$
G_{j}(\omega)=\frac{a}{\pi} \int_{-\pi / 2 a}^{\pi / 2 a} G_{j}(\omega, k) d k,
$$

где $j=0,1$. Перепишем функции (1) и (2) в виде

$$
\begin{gathered}
G_{0}(\omega, k)=\omega \frac{\omega^{2}-t^{2} \Phi(k)}{D^{\prime}(\omega, k)}, \\
G_{1}(\omega, k)=\omega \frac{\omega^{2}-t^{2}[1+\Phi(k)]}{D^{\prime}(\omega, k)},
\end{gathered}
$$

где $D^{\prime}(\omega, k)=\left[\omega^{2}-t^{2} \Phi(k)\right]^{2}-\omega^{2} t^{2}$. Положим $D^{\prime}(\omega, k)=$ $=D_{+}^{\prime}(\omega, k) D_{-}^{\prime}(\omega, k)$, где $D_{ \pm}^{\prime}(\omega, k)=\omega^{2}-t^{2} \Phi(k) \pm t \omega$. Теперь с учетом тождеств

$$
\begin{gathered}
\frac{1}{D^{\prime}(\omega, k)}=\frac{1}{2\left(\omega^{2}-t^{2}(k)\right)} \Sigma^{+}(\omega, k), \\
\frac{1}{D^{\prime}(\omega, k)}=\frac{1}{2 t \omega} \Sigma^{-}(\omega, k),
\end{gathered}
$$

где

$$
\Sigma^{ \pm}(\omega, k)=\frac{1}{D_{-}^{\prime}(\omega, k)} \pm \frac{1}{D_{+}^{\prime}(\omega, k)},
$$

можно выполнить интегрирование (5). Введем вспомогательные интегралы

$$
I_{ \pm}(\omega)=\frac{a}{\pi} \int_{-\pi / 2 a}^{\pi / 2 a} \frac{d k}{D_{ \pm}^{\prime}(\omega, k)}
$$

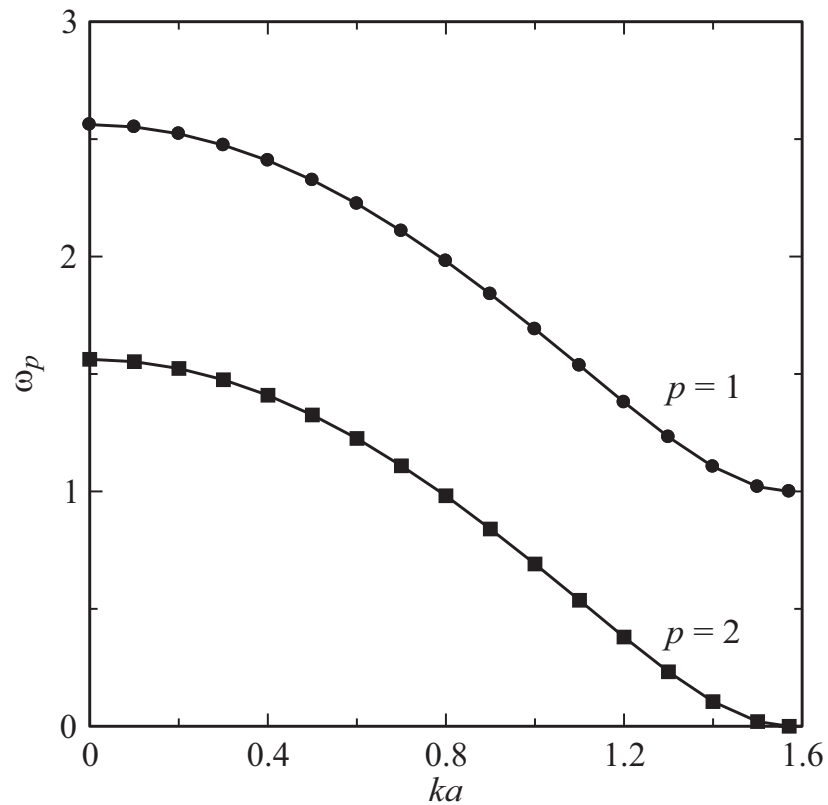

Рис. 2. Энергетические зоны $\omega_{\sigma p}(k)$. На рисунке представлены только зоны, отвечающие положительной энергии. Энергия измеряется в ед. $t$.

и функции $d_{ \pm}=\omega^{2}-2 t^{2} \pm t \omega$. Воспользовавшись справочником [11], получим

$$
I_{ \pm}(\omega)= \begin{cases}\frac{1}{\sqrt{d_{ \pm}^{2}-4 t^{4}},} & d_{ \pm}^{2}>4 t^{4}, \\ \frac{-i}{\sqrt{4 t^{4}-d_{ \pm}^{2}}}, & d_{ \pm}^{2}<4 t^{4} .\end{cases}
$$

Имеем таким образом

$$
\begin{gathered}
G_{0}(\omega)=\frac{\omega}{2}\left(I_{-}(\omega)+I_{+}(\omega)\right), \\
G_{1}(\omega)=G_{0}(\omega)+\frac{t}{2}\left(I_{+}(\omega)-I_{-}(\omega)\right) .
\end{gathered}
$$

Так как плотности состояний $\rho_{j}(\omega)=-\pi^{-1} \operatorname{Im} G_{j}(\omega)$, получим

$$
\begin{gathered}
\rho_{0}(\omega)=\operatorname{sgn}(\omega) \frac{\omega A^{+}(\omega)}{2 \pi}, \\
\rho_{1}(\omega)=\rho_{0}(\omega)+\operatorname{sgn}(\omega) \frac{t A^{-}(\omega)}{2 \pi},
\end{gathered}
$$

где

$$
\begin{gathered}
A^{ \pm}(\omega)=\Theta_{+}(\omega) R_{+}^{-1}(\omega) \pm \Theta_{-}(\omega) R_{-}^{-1}(\omega), \\
R_{ \pm}(\omega)=\sqrt{4 t^{4}-d_{ \pm}^{2}}, \quad \Theta_{ \pm}(\omega) \equiv \Theta\left(4 t^{4}-d_{ \pm}^{2}\right)
\end{gathered}
$$

и $\Theta(\ldots)$ - функция Хэвисайда. Графики функций $\rho_{j}(\omega)$ представлены на рис. 3 для положительной полуоси энергии. Следует отметить, что главное различие плотностей состояний $\rho_{0}(\omega)$ и $\rho_{1}(\omega)$ проявляется при малых энергиях, т.е. в окрестности точки Дирака, где 


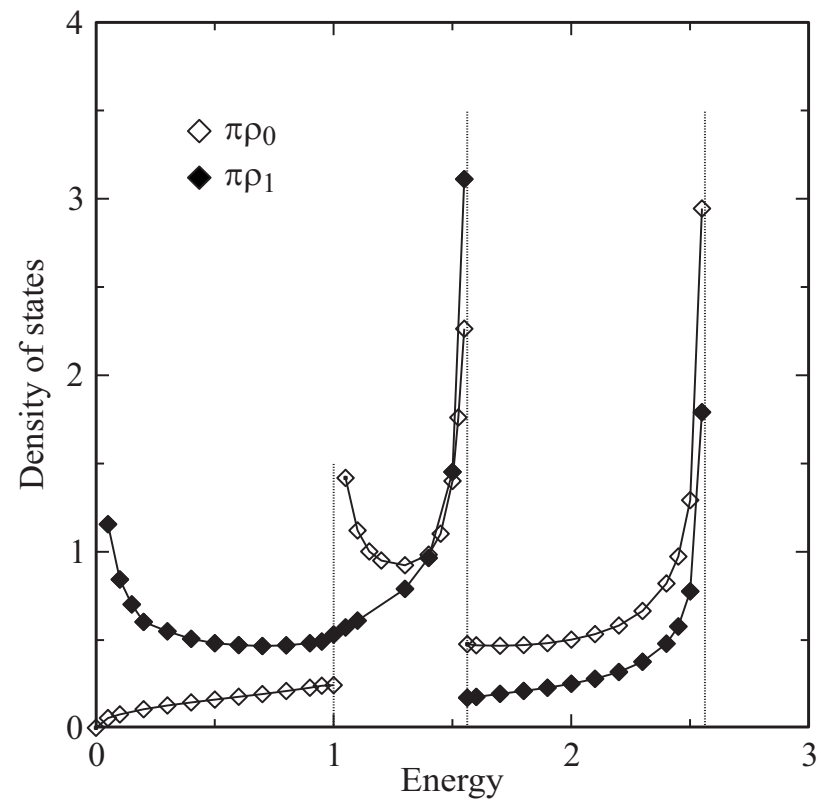

Рис. 3. Плотности состояний $\rho_{0}(\omega)$ (светлые ромбы) и $\rho_{1}(\omega)$ (темные ромбы). Изображены только правые половины симметричных относительно $\omega=0$ функций $\rho_{0}(\omega)$ и $\rho_{1}(\omega)$. Штриховые линии соответствуют асимптотам. Все энергетические величины измеряются в ед. $t$, плотности состояний в ед. $t^{-1}$.

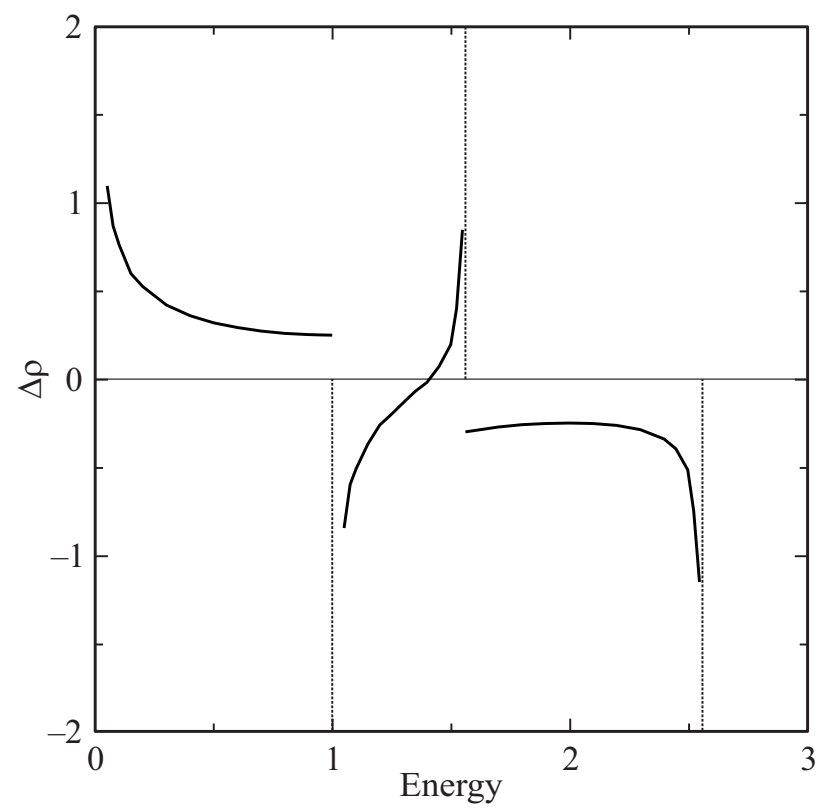

Рис. 4. Функция $\Delta \rho(\omega)=\rho_{1}(\omega)-\rho_{0}(\omega)$. Штриховые линии соответствуют асимптотам. Все энергетические величины измеряются в ед. $t$, плотности состояний - в ед. $t^{-1}$.

$\rho_{0}(\omega) \rightarrow 0$, а $\rho_{1}(\omega) \rightarrow \infty$. Далее, при $\omega \rightarrow t+0^{+}$имеем $\rho_{0}(\omega) \rightarrow \infty$ и $\rho_{1}(\omega) \rightarrow$ const. В интервале энергий от $t(\sqrt{17}-1) / 2$ до $t(\sqrt{17}+1) / 2$ различия чисто количественные. Таким образом, с ростом энергии расхождения между плотностями состояний $\rho_{0}(\omega)$ и $\rho_{1}(\omega)$ сглажива- ются. Интересно также сравнить приведенные на рис. 3 графики с приближенными зависимостями $\rho_{j}(\omega)$, представленными на рис. 3, $a$ работы $[10](\tau=0$, светлые символы). Основное отличие имеет место при $\omega=t$, где в результате использованного в [10] упрощения приближенная функция $\rho_{j}(\omega)$ обращается в нуль.

\section{3. Числа заполнения}

При нулевой температуре числа заполнения атомов $j=0,1$ равны

$$
n_{j}\left(E_{\mathrm{F}}\right)=2 \int_{-\omega_{1}(0)}^{E_{\mathrm{F}}} \rho_{j}(\omega) d \omega
$$

где $E_{\mathrm{F}}-$ уровень Ферми, $-\omega_{1}(0)-$ нижняя граница сплошного спектра, множитель 2 отвечает двум спиновым состояниям. Полагая $E_{\mathrm{F}}=0$ и исходя из сохранения заряда, получим равенство $n_{0}(0)+n_{1}(0)=2$. С учетом (11) и (12) для разности $\Delta n(0)=n_{1}(0)-n_{0}(0)$ найдем выражение

$$
\Delta n(0)=\frac{t}{\pi} \int_{0}^{\omega_{1}(0)} A^{-}(\omega) d \omega
$$

которое легко преобразовать к виду

$$
\Delta n(0)=\frac{t}{\pi}\left[S_{-}(0)-S_{+}(0)\right],
$$

где

$$
S_{-}(0)=\int_{\omega_{1}(\pi / 2 a)}^{\omega_{1}(0)} \frac{d \omega}{R_{-}(\omega)}, \quad S_{+}(0)=\int_{0}^{\omega_{2}(0)} \frac{d \omega}{R_{+}(\omega)} .
$$

Интегрируя, находим $S_{-}(0)=S_{+}(0)=2 K(\eta) / \omega_{1}(0)$, где $K(\eta)$ - полный эллиптический интеграл 1-го рода, $\eta=\omega_{2}(0) / \omega_{1}(0) \quad[11,12]$. Имеем, таким образом, равенство $n_{0}(0)=n_{1}(0)=1$. Полученный результат иллюстрирует рис. 4, где изображена функция $\Delta \rho(\omega)=\rho_{1}(\omega)-\rho_{0}(\omega)$, проявляющая определенную антисимметрию, в силу чего $\int_{0}^{\omega_{1}(0)} \Delta \rho(\omega) d \omega=0$.

Тот же результат можно получить и непосредственно. Вычислим значение числа заполнения $n_{0}(0)=\left[J_{-}(0)+J_{+}(0)\right] / \pi$, где

$$
J_{-}(0)=\int_{\omega_{1}(\pi / 2 a)}^{\omega_{1}(0)} \frac{\omega d \omega}{R_{-}(\omega)}, \quad J_{+}(0)=\int_{0}^{\omega_{2}(0)} \frac{\omega d \omega}{R_{+}(\omega)}
$$

Воспользовавшись [11], можно показать, что

$$
\begin{gathered}
J_{-}(0)=2[(1+\eta) \Pi(-\eta, \eta)-\eta K(\eta)], \\
J_{+}(0)=2[(1+\eta) \Pi(-\eta, \eta)-K(\eta)],
\end{gathered}
$$


где П $(\eta, \eta)$ - полный эллиптический интеграл 3-го рода. Тогда имеем

$$
n_{0}(0)=\frac{2(1+\eta)}{\pi}[2 \Pi(-\eta, \eta)-K(\eta)] .
$$

Так как, согласно [12],

$$
\Pi(-\eta, \eta)=\frac{1}{4(1+\eta)}[\pi+2(1+\eta) K(\eta)],
$$

получаем $n_{0}(0)=1$.

Представим теперь, что можно определенным образом смещать уровень Ферми относительно точки Дирака (например, приложением электрического поля между подложкой и нанолентой по схеме FET). Тогда можно получить зависимости $n_{j}\left(E_{\mathrm{F}}\right)=1+v_{j}\left(E_{\mathrm{F}}\right)$, где $v_{j}\left(E_{\mathrm{F}}\right)>0$ и $v_{j}\left(E_{\mathrm{F}}\right)<0$ при положительных и отрицательных значениях $E_{\mathrm{F}}$, причем $v_{j}\left(-E_{\mathrm{F}}\right)=-v_{j}\left(E_{\mathrm{F}}\right)$. С учетом (11) и выражений для $S_{ \pm}\left(E_{\mathrm{F}}\right)$ и $J_{ \pm}\left(E_{\mathrm{F}}\right)$, приведенных в Приложении, получим

$$
\begin{gathered}
v_{0}\left(E_{\mathrm{F}}\right)=\left[J_{+}\left(E_{\mathrm{F}}\right)+J_{-}\left(E_{\mathrm{F}}\right)\right] \pi, \\
v_{1}\left(E_{\mathrm{F}}\right)=v_{0}\left(E_{\mathrm{F}}\right)+t\left[S_{-}\left(E_{\mathrm{F}}\right)-S_{+}\left(E_{\mathrm{F}}\right)\right] / \pi .
\end{gathered}
$$

Легко показать, что $v_{0}(0)=v_{1}(0)=0$. Не проводя численных расчетов, а лишь учтя, что значения $v_{j}\left(E_{\mathrm{F}}\right)$ определяются площадями под кривыми $\rho_{j}\left(E_{\mathrm{F}}\right)$, по рис. 3 можно проследить качественный характер зависимостей $v_{j}$ от $E_{\mathrm{F}}$, соответствующим образом сдвигая уровень Ферми.

\section{4. Точечный дефект в графеновой наноленте}

В качестве примера применения полученных результатов рассмотрим задачу о точечном дефекте (примеси замещения, вакансии) в графеновой наноленте. Функции Грина (10) позволяют найти точное решение этой задачи в рамках модели Костера-Слэтера $[13,14]$. Отметим, что в работе [15] эта модель была применена к описанию примеси замещения в бесконечном листе графена.

Будем считать, что возмущение $V$, вносимое дефектом, локализовано только в одном узле решетки наноленты. Если дефект занимает узел, в котором изначально находился атом 0 (1), то уравнение, определяющее энергию наводимого этим дефектом локального уровня, имеет вид $V \operatorname{Re} G_{0(1)}(\omega)=1$, где $|\omega|>\omega_{1}(0)$. С учетом (10) это уравнение можно представить в виде

$$
L_{0(1)}(\omega)=V^{-1},
$$

где

$$
\begin{gathered}
L_{0}(\omega)=\frac{\omega}{2}\left(\frac{1}{R_{+}(\omega)}+\frac{1}{R_{-}(\omega)}\right), \\
L_{1}(\omega)=L_{0}+\frac{t}{2}\left(\frac{1}{R_{+}(\omega)}-\frac{1}{R_{-}(\omega)}\right) .
\end{gathered}
$$

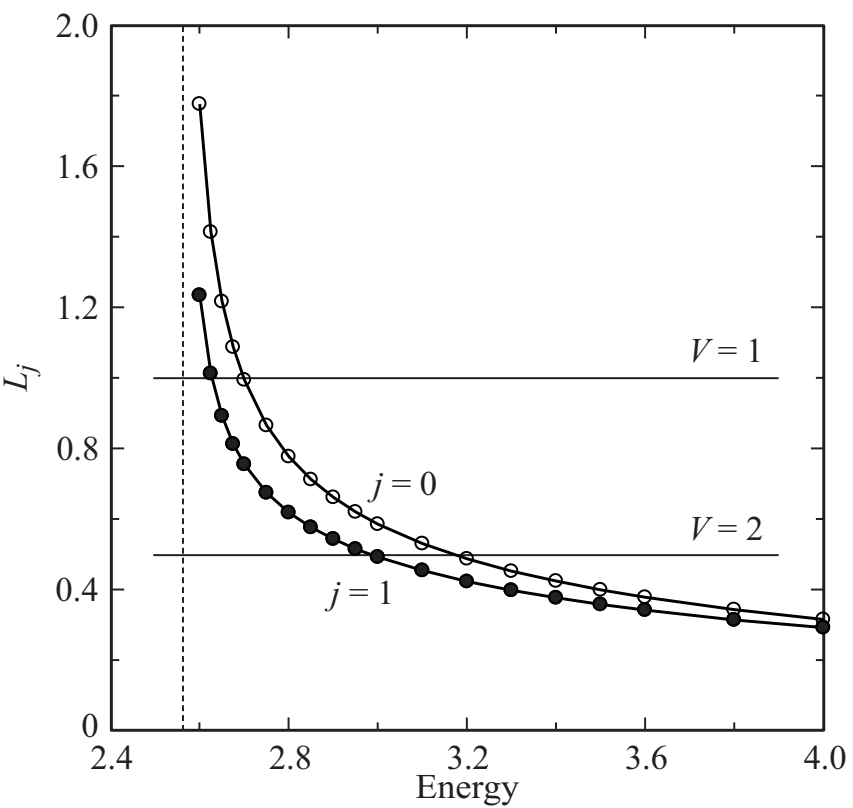

Рис. 5. Схема решения уравнении (20). Точки пересечения тонких горизонтальных линий, отвечающих значениям $V^{-1}$ (на рисунке $V=t$ и $2 t)$, с графиками $L_{0}(\omega)$ и $L_{1}(\omega)$, определяют энергии локальных уровней $\varepsilon_{0}^{*}$ и $\varepsilon_{1}^{*}$, лежащих (при $V>0$ ) выше верхнего края сплошного спектра. Штриховая линия соответствует асимптоте при $\omega=\omega_{1}(0)$. Все энергетические величины измеряются в ед. $t$.

Схема решения уравнений (20) при $V>0$ представлена на рис. 5. Локальные уровни $\varepsilon_{0}^{*}$ и $\varepsilon_{1}^{*}$, определяемые точками пересечения горизонтальной линии, соответствующей значению $V^{-1}$, с функциями $L_{0}(\omega)$ и $L_{1}(\omega)$, расположены выше верхнего края сплошного спектра наноленты. Из рис. 5 следует, что $\varepsilon_{0}^{*}>\varepsilon_{1}^{*}$. Действительно, полагая $V / t \ll 1$ и $\varepsilon_{j}^{*}=\omega_{1}(0)+\xi_{j}$, где $\xi_{j} \ll 1$, получим $\xi_{0} \approx(V / 4 t)^{2}\left[\omega_{1}^{2}(0) /\left(2 \omega_{1}(0)-t\right)\right]$ и $\xi_{1} \approx(V / 4 t)^{2}\left[\left(\omega_{1}(0)-t\right)^{2} /\left(2 \omega_{1}(0)-t\right)\right]$, так что $\xi_{0}>\xi_{1}$ и $\xi_{j} \propto(V / t)^{2}$. В пределе $V / t \gg 1$ имеем $\varepsilon_{0}^{*} \approx \varepsilon_{1}^{*} \approx V$. При $V<0$ уровни $\varepsilon_{0}^{*}$ и $\varepsilon_{1}^{*}$ лежат ниже нижнего края сплошного спектра. Более того, так как $L_{j}(\omega)=-L_{j}(-\omega)$, то при смене знака возмущения $V$ энергии уровней $\varepsilon_{j}^{*}$ также меняют знак.

Воспользовавшись результатами работы [15], при нулевой температуре для чисел заполнения локальных уровней $n_{j}^{*}$ получим

$$
n_{j}^{*} \approx 2 c_{j} \Theta\left(E_{\mathrm{F}}-\varepsilon_{j}^{*}\right), \quad c_{j}=\frac{1}{V^{2}\left|\partial L_{j}(\omega) / \partial \omega\right|_{\varepsilon_{j}^{*}}},
$$

где $c_{j}-$ весовой множитель заполнения локального уровня с энергией $\varepsilon_{j}^{*}$. Дифференцируя функции (21), получим

$$
\begin{aligned}
\frac{\partial L_{0}(\omega)}{\partial \omega}= & \frac{1}{2 R_{+}(\omega)}\left(1-\frac{\omega(2 \omega+t) d_{+}}{R_{+}^{2}}\right) \\
& +\frac{1}{2 R_{-}(\omega)}\left(1-\frac{\omega(2 \omega-t) d_{-}}{R_{-}^{2}}\right),
\end{aligned}
$$




$$
\frac{\partial L_{1}(\omega)}{\partial \omega}=\frac{\partial L_{0}(\omega)}{\partial \omega}+\frac{t(2 \omega-t) d_{-}}{R_{-}^{3}(\omega)}-\frac{t(2 \omega+t) d_{+}}{R_{+}^{3}(\omega)} .
$$

Отсюда следует, что $c_{j} \sim 0$ при $|V| / t \ll 1$ и $c_{j} \sim 1$ при $|V| / t \gg 1$. Так как $\left|\varepsilon_{0}^{*}\right|>\left|\varepsilon_{1}^{*}\right|$, имеем $c_{0}>c_{1}$.

\section{5. Заключение}

Итак, в настоящей работе нам удалось получить точные аналитические выражения не только для энергетических плотностей состояний $\rho_{j}(\omega)$, но и для чисел заполнения $n_{j}$ двух типов атомов углерода $j=0,1$ зигзагообразной кромки графеновой наноленты. Способствовали этому два обстоятельства: простая структурная модель (рис. 1) и представление функций Грина в виде (10). Для случая, когда уровень Ферми $E_{\mathrm{F}}$ совпадает с принятой за нуль энергии точкой Дирака (недопированная нанолента), получаем $n_{j}=1$. Зависимость $n_{j}\left(E_{\mathrm{F}}\right)$ удалось представить в виде неполных табулированного интеграла 1-го рода $\mathrm{F}(\mu, \eta)$ (см., например, [16]) и нетабулированного интеграла 3-го рода $\Pi(\mu,-\eta, \eta)$, алгоритмы вычисления которого известны (см., например, [17] и приведенные там ссылки).

В качестве примера применения полученных результатов рассмотрена задача о локальном дефекте замещения в модели Костера-Слэтера. Показано, что предложенный в настоящей работе подход позволяет точно решить эту задачу. Аналогичным образом можно рассмотреть задачи об адсорбированном атоме и декорирующей частице, возникающие при изучении функционализации графеновых нанообъектов.

\section{Приложсние}

Введем интегралы

$S_{ \pm}\left(E_{\mathrm{F}}\right)=\int_{0}^{E_{\mathrm{F}}} \Theta_{ \pm}(\omega) \frac{d \omega}{R_{ \pm}(\omega)}, J_{ \pm}\left(E_{\mathrm{F}}\right)=\int_{0}^{E_{\mathrm{F}}} \Theta_{ \pm}(\omega) \frac{\omega d \omega}{R_{ \pm}(\omega)}$.

Обозначая неполные интегралы 1-го и 3-го рода как $\mathrm{F}\left(\mu_{ \pm}, \eta\right)$ и $\Pi\left(\mu_{ \pm},-\eta, \eta\right)$, с помощью формулы 3.147 и 3.148 справочника [11] получим следующие выражения:

$$
\begin{gathered}
S_{+}\left(0 \leq E_{\mathrm{F}}<\omega_{2}(0)\right)=S_{+}(0)-\frac{2}{\omega_{1}(0)} \mathrm{F}\left(\mu_{+}, \eta\right), \\
S_{+}\left(E_{\mathrm{F}} \geq \omega_{2}(0)\right)=S_{+}(0), \\
J_{+}\left(0 \leq E_{\mathrm{F}}<\omega_{2}(0)\right)=J_{+}(0)-2[(1+\eta) \\
\left.\times \Pi\left(\mu_{+},-\eta, \eta\right)-\mathrm{F}\left(\mu_{+}, \eta\right)\right], \quad J_{+}\left(E_{\mathrm{F}} \geq \omega_{2}(0)\right)=J_{+}(0),
\end{gathered}
$$

где

$$
\begin{gathered}
\mu_{+}\left(0 \leq E_{\mathrm{F}}<\omega_{2}(0)\right)=\arcsin \sqrt{\frac{1-E_{\mathrm{F}} / \omega_{2}(0)}{1+E_{\mathrm{F}} / \omega_{1}(0)}}, \\
\mu_{+}\left(E_{\mathrm{F}}>\omega_{2}(0)\right)=0
\end{gathered}
$$

$$
\begin{gathered}
S_{-}\left(0 \leq E_{\mathrm{F}}<t\right)=0, \\
S_{-}\left[t \leq E_{\mathrm{F}} \leq \omega_{1}(0)\right]=S_{-}(0)-\frac{2}{\omega_{1}(0)} \mathrm{F}\left(\mu_{-}, \eta\right), \\
J_{-}\left(0 \leq E_{\mathrm{F}}<t\right)=0, \\
J_{-}\left[t \leq E_{\mathrm{F}} \leq \omega_{1}(0)\right]=J_{-}(0)-2\left[(1+\eta) \Pi\left(\mu_{-},-\eta, \eta\right)\right. \\
\left.-\eta \mathrm{F}\left(\mu_{-}, \eta\right)\right],
\end{gathered}
$$

где $\mu_{-}\left(0 \leq E_{\mathrm{F}}<t\right)=0$,

$$
\mu_{-}\left(t \leq E_{\mathrm{F}} \leq \omega_{1}(0)\right)=\arcsin \left(\eta^{-1} \sqrt{\frac{1-E_{\mathrm{F}} / \omega_{1}(0)}{1+E_{\mathrm{F}} / \omega_{2}(0)}}\right) .
$$

\section{Конфликт интересов}

Авторы заявляют, что у них нет конфликта интересов.

\section{Список литературы}

[1] R. Taira, A. Yamanaka, S. Okada. Appl. Phys. Express, 9, 115102 (2016).

[2] M.R. Mananghaya, G.N. Santos, D. Yu, C. Stampfl. Sci. Rep., 7, 15727 (2017).

[3] K. Nakada, M. Fujita, G. Dresselhaus, M. Dresselhaus. Phys. Rev. B, 54, 17954 (1996).

[4] A.H. Castro Neto, F. Guinea, N.M.R. Peres, K.S. Novoselov, A.K. Geim. Rev. Mod. Phys., 81, 109 (2009).

[5] D.S.L. Abergel, V. Apalkov, J. Berashevich, K. Ziegler, T. Chakraborty. Adv. Phys., 59, 261 (2010).

[6] V.N. Kotov, B. Uchoa, V.M. Pereira, A.H. Castro Neto, F. Guinea. Rev. Mod. Phys., 84, 1067 (2012).

[7] Ф. Андерсон. УФН, 127, 19 (1979).

[8] J. Cserti. Am. J. Phys., 68, 896 (2000).

[9] G. Jose, R. Malla, V. Srinivasan, A. Sharma, S. Gangadharaiah. arXiv: $1711.08204 \mathrm{v} .1$.

[10] С.Ю. Давыдов. ФТТ, 61, 610 (2019).

[11] И.С. Градштейн, И.М. Рыжик. Таблищы интегралов, сумм, рядов и произведений (М., Наука, 1971).

[12] P.F. Bird, M.D. Friedman. Handbook of Elliptic integrals for Engineers and Scientists (Berlin-Heidelberg-N.Y., Springer, 1971).

[13] Ю.А. Изюмов, М.В. Медведев. Теория магнитоупорядоченных кристаллов с примесями (М., Наука, 1970).

[14] С.Ю. Давыдов, А.А. Лебедев, О.В. Посредник. Элементарное введение в теорию наносистем (СПб., Лань, 2014).

[15] С.Ю. Давыдов. ФТП, 50, 816 (2016).

[16] Е. Янке, Ф. Эмде, Ф. Лёш. Специальные функции (М., Наука, 1977).

[17] T. Fukushima. IEEE 22nd Symp. on Computer Arithmetic (ARITH) (2015) p. 50.

Редактор Г.А. Оганесян 


\section{Exactly solvable model of graphene nanoribbon with the zigzag edges}

S.Yu. Davydov ${ }^{1}$, A.V. Zubov ${ }^{2}$

${ }^{1}$ loffe Institute,

194021 St. Petersburg, Russia

2 St. Petersburg National Research University

of Information Technologies, Mechanics and Optics

197101 St. Petersburg, Russia

Abstract Exact analytical expressions for the local densities of states and occupation numbers for atoms of graphene nanoribbon with zigzag edges are obtained in the scope of simple structural model. As an example of obtaining results the problem on the Koster-Slater defect is considered. 